\title{
Desenvolvimento de software para a organização da informação de um serviço de saúde ocupacional hospitalar
}

Software development for data processing in a hospital occupational health service

Desarrollo de software para ordenamiento de las informaciones en un servicio de salud

Maria Cláudia Parro ${ }^{I}$, Yolanda Dora Martinez Évora ${ }^{\mathrm{II}}$

\section{RESUMO}

A tecnologia da informação representa uma ferramenta que pode disponibilizar maior facilidade na realização dos trabalhos e incrementar a assistência prestada. O objetivo deste estudo foi desenvolver um software para a organização dos dados de acidente de trabalho com material biológico, caracterização epidemiológica e monitoramento da evolução clínica da população, visando o planejamento da assistência de enfermagem em Saúde Ocupacional. A metodologia compreendeu as fases de definição do projeto; definição dos requisitos e desenvolvimento do sistema. O software foi desenvolvido em linguagem DELPHI 7 para funcionar em plataforma Microsoft \& Windows na versão "XP" 2003, tendo como base de armazenamento de dados o Firebird 2.0. O sistema informatizado foi estruturado em três módulos de cadastro e o módulo SINAN. Acredita-se que esse produto inovador auxiliará o enfermeiro na tomada de decisão e facilitará o planejamento e acompanhamento de enfermagem nos casos de acidente com material biológico até o seu desfecho.

Descritores: Software; Saúde do Trabalhador; Enfermagem; Tomada de Decisões.

\section{ABSTRACT}

Information technology is a tool that can provide better performing in the work and improve care. The purpose of this study was to develop a software program to enable data processing of work-related accidents involving hazardous biological material, epidemiological characterization, and clinical evolution monitoring of the population, aiming the Occupational Health Nursing Care planning. The methodology was composed by the phases of the project definition, requirements definition and system development. The software was developed in Delphi 7 language to run on Microsoft Windows platform - "XP" 2003, having the Firebird 2.0 as base of data storage. The system was structured in three modules of register, and the SINAM module. It is believed that this innovative product will assist nurses in decision-making and will facilitate the planning and monitoring of nursing care in cases of accidents involving hazardous biological material.

Descriptors: Software; Occupational Health; Nursing; Decision Making.

\section{RESUMEN}

La tecnología de la información es una herramienta que puede proporcionar una mayor facilidad en el desempeño de la labor y mejorar la atención. El objetivo deste estudio fue desarrollar un software que haga posible el ordenamiento de los datos de accidentes de trabajo con material biológico, caracterización epidemiológica y monitoreo de evolución clínica de población, con vistas en planeamiento de la asistencia en Salud Ocupacional. La metodología empleada comprende las fases de la definición del proyecto, la definición de los requisitos y el desarrollo del sistema. El software fue desarrollado en lenguaje Delphi 7 para ejecutarse en Microsoft Windows - plataforma "XP" 2003, basado en el almacenamiento de datos Firebird 2.0. El sistema se estructura en tres módulos, y el módulo de registro SINAN. Se cree que este innovador producto para ayudar a las enfermeras en la toma de decisiones y facilitar la planificación y el seguimiento de enfermería en casos de accidentes con material biológico.

Descriptores: Programas Informáticos; Salud Laboral; Enfermería; Toma de Decisiones.

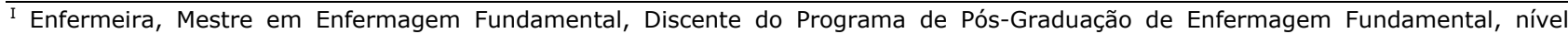
Doutorado, Escola de Enfermagem de Ribeirão Preto (EERP), Universidade de São Paulo (USP). Docente, Universidade Paulista. Ribeirão Preto, SP, Brasil. E-mail: mcparro@bol.com.br.

II Enfermeira, Doutora em Enfermagem, Livre-docente. Professora Titular, EERP, USP. Ribeirão Preto, SP, Brasil. E-mail: yolanda@eerp.usp.br.
} 


\section{INTRODUÇÃO}

Os sistemas de informação tornaram-se uma das principais tecnologias computacionais capazes de propagar de maneira rápida, fácil e segura as informações necessárias para mediar a aprendizagem e auxiliar os profissionais no processo de trabalho. Estes, enquanto instrumentos de trabalho na saúde, são importantes recursos de apoio às ações administrativo burocráticas e àquelas ancoradas em conhecimentos técnico-científicos, sobretudo as que dependem de informações atualizadas ${ }^{(1)}$.

A tecnologia da informação representa, portanto, uma ferramenta que pode disponibilizar maior facilidade na realização dos trabalhos e incrementar a assistência prestada. O computador no cotidiano da enfermagem tem o intuito de auxiliar o enfermeiro a organizar e administrar uma quantidade excessiva de informações proporcionando em tempo real, todo e qualquer dado que necessita para o desenvolvimento de suas ações ${ }^{(2)}$.

O fator chave para a deficiente utilização dos dados na geração de informação é a falta de sistemas informatizados para processar dados e disponibilizar informações em um formato que seja facilmente compreendido pelas pessoas. O tempo despendido na recuperação das diversas informações do cuidado prestado ao paciente em sistema de registro manual compromete, sobremaneira, o processo de tomada de decisão.

Apesar do uso da informática na área da saúde e da enfermagem encontrar-se em franco desenvolvimento, tanto nacional quanto internacionalmente, observa-se, ainda, em muitas instituições hospitalares brasileiras, a inexistência de registro sistematizado de dados para gerar informações que orientem as medidas a serem tomadas pelo enfermeiro, diante de um acidente de trabalho com material perfurocortante ou biológico.

Os trabalhadores de saúde, em especial os de enfermagem, que representam a maior força de trabalho presente nas instituições de saúde, desenvolvem atividades em ambiente de trabalho onde estão expostos a riscos ocupacionais peculiares às atividades que executam na assistência a pacientes, famílias e comunidade. Estes riscos são classificados em: biológicos, físicos, químicos, psicossocial e fatores ergonômicos ${ }^{(3-6)}$.

Ao se analisar o risco biológico, conclui-se que é um dos principais geradores de periculosidade e insalubridade para os trabalhadores de enfermagem, em decorrência do contato, direto e permanente, dos profissionais com pacientes e objetos contaminados por patógenos responsáveis por doenças como a Hepatite e a Síndrome da Imunodeficiência Adquirida - $\operatorname{AIDS}^{(7)}$.

Para muitos profissionais da saúde, é um desafio trabalhar com sistemas informatizados, muitas vezes por não terem intimidade com a máquina e até por comodismo, preferem ficar com um prontuário manual e depois arquivar em uma sala, ocupando espaço ${ }^{(8)}$.

Frente aos desafios apresentados pelo dinamismo e complexidade dos problemas nesse campo, os profissionais de enfermagem estão sendo solicitados a demonstrar novas competências, além daquelas necessárias à execução de suas atividades profissionais, para atender transformações sociais e produtivas de trabalho humano. Necessitam, portanto, adquirir uma nova postura quanto à utilização de sistemas informatizados, uma vez que estes vêm para melhorar o rendimento profissional e facilitar as atividades laborais ${ }^{(9-}$ 10).

A confiabilidade e as facilidades quanto à manutenção, ao acesso e à atualização de informações, bem como, quanto às possibilidades de um tratamento estatístico das mesmas, fazem da Informática, por meio de seus softwares, um instrumento indispensável ao gerenciamento do banco de dados e à esquematização dos cadastros propostos no Serviço de Saúde Ocupacional.

A enfermagem, a exemplo de outras profissões, deve utilizar os recursos computacionais para incrementar a produtividade e a qualidade, como forma de agregar e analisar as informações relevantes para a tomada de decisão e para o desempenho eficiente de todas as suas funções ${ }^{(11)}$.

Assim, o desenvolvimento de um software possibilitará não só uma caracterização epidemiológica, mas também um monitoramento da evolução clínica da população visando subsidiar e nortear o planejamento da assistência de enfermagem.

Diante da necessidade de se organizar um Serviço de Saúde Ocupacional Hospitalar, algumas questões emergiram: Como é o registro manual das notificações de acidente no trabalho com material biológico? Como ajudar os enfermeiros a melhorar o processo de documentação dos eventos ocorridos? A implantação de um sistema informatizado facilitará o acompanhamento dos casos notificados?

O objetivo deste estudo foi desenvolver um software para a organização dos dados de acidente de trabalho com material biológico, caracterização epidemiológica e 
monitoramento da evolução clínica da população, visando o planejamento da assistência de enfermagem em Saúde Ocupacional.

\section{PERCURSO METODOLÓGICO}

Trata-se de uma pesquisa de desenvolvimento, baseada na engenharia de software e fundamentada no ciclo de vida de desenvolvimento de sistemas ${ }^{(12-13)}$

O estudo foi realizado durante o ano de 2010 e teve como base o Serviço de Saúde Ocupacional de uma instituição hospitalar filantrópica de médio porte, localizada na região Noroeste do Estado de São Paulo.

O referido Serviço dispõe de arquivo de registro manual e de equipe multidisciplinar em que há a participação de uma enfermeira responsável pelas atividades de assistência ao trabalhador e por atividades de tomada de decisão, de controle e manutenção dos registros, de levantamentos epidemiológicos e tratamentos estatísticos dos dados pertencentes ao Serviço de Saúde Ocupacional. Além disso, cabe à referida enfermeira, repassar ao Serviço Ambulatorial de Especialidade em Moléstias Infecciosas (SAE-MI), as informações referentes aos casos de acidentes com material biológico para que esse serviço proceda ao acompanhamento dos casos.

A estrutura metodológica compreendeu as fases de definição do projeto; definição dos requisitos; e, desenvolvimento do sistema, descritas a seguir:

- Definição do Projeto: Nessa etapa foram definidas as funções do software, o estabelecimento dos recursos necessários à sua elaboração e a definição das estimativas de prazo e custo.

Realizou-se primeiramente o estudo do sistema atual. Nessa fase foi verificado a forma manual de registro das informações, a existência de um Sistema de Apoio à Decisão, o fluxo de dados no processo de notificação do acidente de trabalho com material biológico e a interpretação dos Fluxogramas de Acidente Profissional e de Análise da Situação, utilizados pela instituição para organizar e direcionar a informação relativa à notificação, tomada de decisão e assistência ao acidente com material biológico.

- Definição de requisitos: Essa etapa implicou na coleta dos requisitos especificados pelo pesquisador e discriminados a partir do conjunto mínimo de dados estabelecidos pelo Ministério da Saúde (MS) e disponibilizados por meio do Manual de Exposição a Materiais Biológicos, a saber: Ficha de Notificação de Acidentes Biológicos com Profissionais de Saúde do
Sistema de Informação de Agravos de Notificação (SINAN); Fluxograma de Profilaxia Antirretroviral após Exposição Ocupacional, destinado à avaliação de Quimioprofilaxia (QP) para o HIV.

Diante das especificações dos requisitos, estabeleceram-se os modelos de fluxo e estrutura da informação.

- Desenvolvimento do sistema: O sistema de informação foi desenvolvido em linguagem DELPHI 7, uma vez que esta linguagem proporciona ampla utilização de telas ativas, fornece ao usuário a proteção de dados por meio de senha além de poder ser utilizado em rede. Teve como base de armazenamento de dados o Firebird 2.0, com característica de acesso livre, compatível com aqueles utilizados na instituição em estudo. $O$ sistema foi desenvolvido para funcionar em plataforma Microsoft $\circledR$ Windows na versão "XP" 2003.

Participaram no processo de construção do software um analista de sistemas, um programador e o próprio pesquisador, como enfermeiro especialista em Saúde Ocupacional.

A população de estudo foi constituída pela informação gerada no Serviço de Saúde Ocupacional da instituição em tela, conforme o estabelecido pelo Ministério da Saúde (MS) e disponibilizado por meio do Manual de Exposição a Materiais Biológicos.

A coleta de dados foi realizada pelo próprio pesquisador em dois momentos: por meio de análise documental realizou-se a análise funcional do sistema manual de registro das informações. No segundo momento foram especificados os requisitos do sistema informatizado.

A pesquisa foi submetida ao Comitê de Ética em Pesquisa da Faculdade de Medicina de Catanduva, obtendo parecer favorável ao seu desenvolvimento (Ofício 08/2010). Foram respeitados todos os preceitos ético legais que regem a pesquisa com seres humanos, atendendo a Resolução 196/96 do Conselho Nacional de Saúde ${ }^{(14)}$.

\section{RESULTADOS}

Por meio da análise documental realizou-se, primeiramente, o estudo do sistema em uso. Nessa fase foi verificada a forma manual de registro das informações, a existência de um Sistema de Apoio à Decisão, o fluxo de dados no processo de notificação do acidente de trabalho com material biológico e a 
interpretação dos Fluxogramas de Acidente Profissional e de Análise da Situação, utilizados pela instituição para organizar e direcionar a informação relativa à notificação, tomada de decisão e assistência ao acidente com material biológico.

Uma observação relevante nesta fase da pesquisa diz respeito à ausência do enfermeiro da unidade de Saúde Ocupacional no cenário do acidente em decorrência de sua jornada de trabalho. Nessas situações todo o processo de coleta e análise dos dados do acidente é realizado por enfermeiros assistenciais em esquema de plantão, que ficam responsáveis por registrar, analisar e decidir sobre o caso, ainda que não tenham participado de treinamentos para análise e tomada de decisão adequadas, ou que ainda não apresentem competência técnica para fazê-lo. Tal realidade reflete na imprecisão dos registros e nas condutas a serem implementadas.

A falta de conhecimento do enfermeiro do Serviço Ambulatorial de Especialidade em Moléstias Infecciosas (SAE-MI) em processar os dados em informações é, também, uma realidade. Verificou-se que em diversos casos as fichas de registros de dados no formato manual estavam incompletas, além das informações, por vezes, serem mal interpretadas, comprometendo assim, o diagnóstico e as intervenções cabíveis na assistência imediata e no acompanhamento do caso.

Diante dos problemas identificados, estabeleceramse os modelos de fluxo e estrutura da informação. O software foi elaborado conforme os seguintes requisitos: desenhado e construído de forma a facilitar a entrada e manipulação da informação; suficientemente flexível para permitir a modificação do formato de telas e de entrada de dados, caso se faça necessário; permitir a entrada e a recuperação de dados de uma forma mais eficiente do que o sistema manual existente; permitir entrada de texto livre; desenhado para fornecer uma rápida entrada de dados, utilizando-se de terminais de fácil acesso a todos os usuários; permitir que os planos de cuidados sejam utilizados quando necessários, sem destruir as informações anteriores.

A etapa de desenvolvimento do software traduziu o conjunto de requisitos em um modelo informatizado, estruturado em três módulos de cadastro (Funcionários, Empresas Contratantes e Município) e o módulo de Sistema de Informação de Agravos de Notificação (SINAN).

A tela inicial do software possui as opções:

Cadastro e Lista que abrem acesso à realização dos cadastramentos necessários e a uma base de dados destinada à consulta das informações e controle da assistência prestada.

A opção Lista disponibiliza dois bancos de dados: um com informações sobre os atendimentos e exames laboratoriais realizados em circunstâncias de acidente com material biológico e outro com informações técnicas (administração, efeitos adversos e interações) referentes aos medicamentos utilizados na quimioprofilaxia dos acidentes com risco de contaminação (Figura 1). 
Figura 1: Tela Efeitos Adversos e Interações Medicamentosas. Software para organização das informações de um serviço de Saúde Ocupacional. Caranduva, SP, 2010.

\begin{tabular}{|c|c|c|c|c|c|c|}
\hline \multicolumn{7}{|c|}{ Efeitos Adversos e Interaçốes dos Medicamentos Utilizados na Quimioprofilaxia } \\
\hline$\underset{\text { Inserit }}{\square}$ & 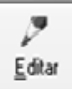 & $\begin{array}{c}3 \\
\text { Excluir }\end{array}$ & $\begin{array}{c}\text { 圆 } \\
\text { salvar }\end{array}$ & $\underset{\text { Cancelar }}{X}$ & $\underset{\text { Eechst }}{\text { Y }}$ & \\
\hline \multicolumn{7}{|c|}{ Código: } \\
\hline \multicolumn{7}{|c|}{8} \\
\hline \multicolumn{6}{|c|}{ Medicamento: } & Interaç̄es: \\
\hline \multirow{2}{*}{\multicolumn{6}{|c|}{ 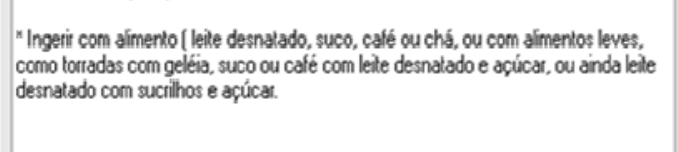 }} & \multirow{6}{*}{ 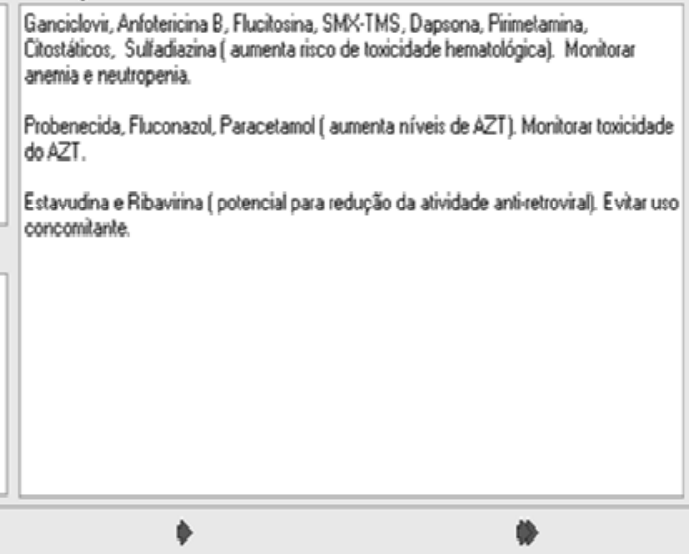 } \\
\hline & & & & & & \\
\hline & & & & & & \\
\hline \multicolumn{6}{|c|}{ Efeitos Adversos: } & \\
\hline \multicolumn{6}{|c|}{ 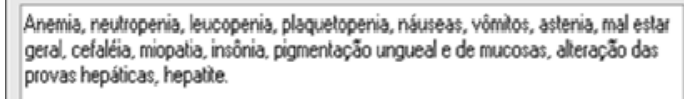 } & \\
\hline \multicolumn{5}{|c|}{ B } & & \\
\hline
\end{tabular}

O módulo Cadastro de Funcionários (Figura 2) proporciona o cadastramento de funcionários criando um banco de dados para organização e acessibilidade das informações relativas aos funcionários atendidos pelo serviço de Saúde Ocupacional nas situações de acidente.
Para minimizar as atividades de digitação o campo envolvendo data de nascimento, dispõe de um calendário que permite a adição dos dados com um toque no dispositivo de entrada (mouse).

Figura 2: Tela Cadastro de Funcionário. Software para organização das informações de um serviço de Saúde Ocupacional. Caranduva, SP, 2010.

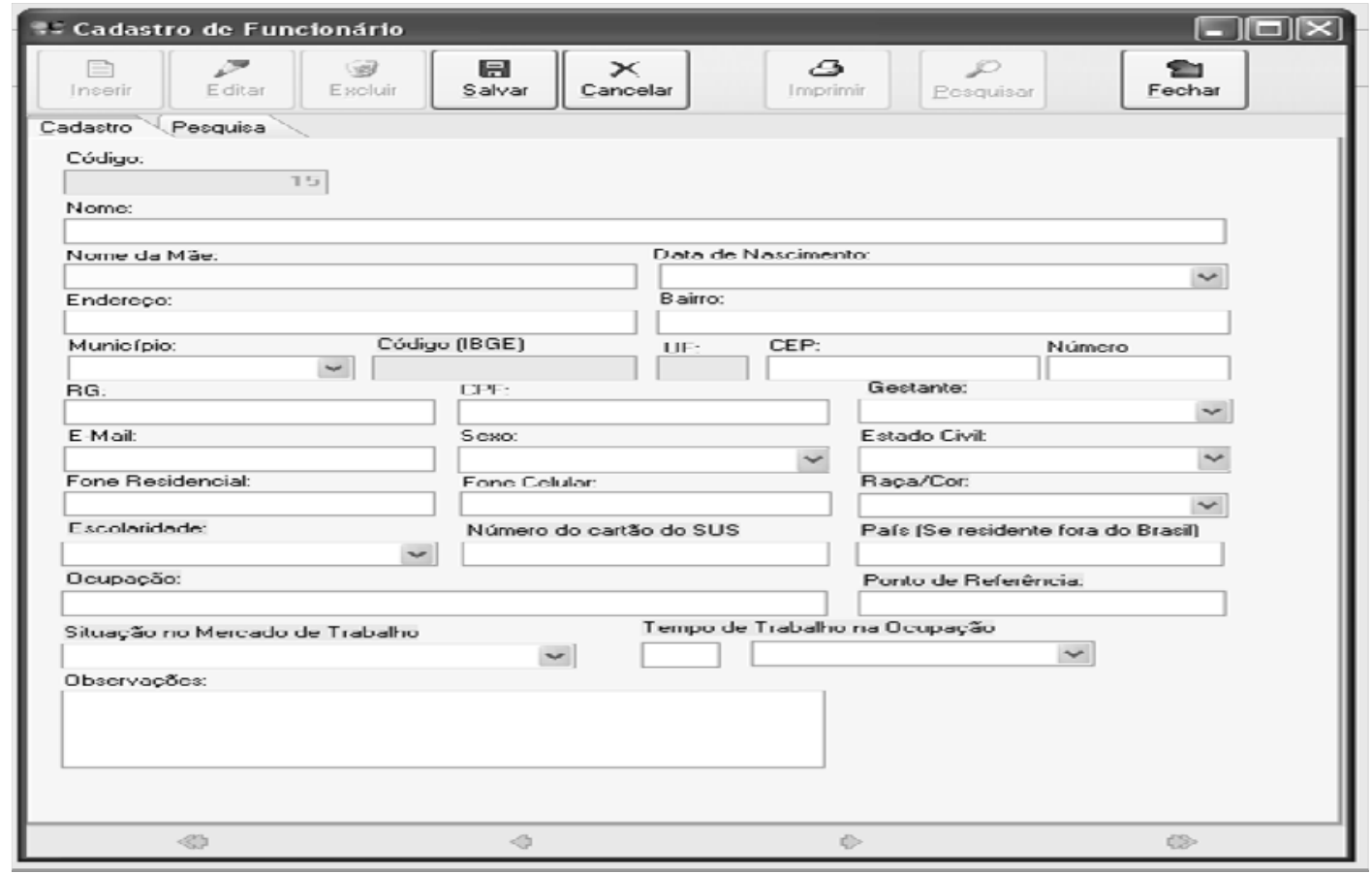


Os campos: município, gestante, sexo, estado civil, raça e cor, escolaridade, situação no mercado de trabalho e tempo de trabalho também poderão ser cadastrados com toque no dispositivo de entrada (mouse). Após a inserção de todos os campos, torna-se obrigatório salvar os dados cadastrados.

O acesso à interface Pesquisa dos Funcionários Cadastrados tem a finalidade de agilizar o processo de busca dos dados de funcionários e minimizar as atividades de digitação do usuário no cadastramento das notificações dos acidentes de trabalho.

O módulo Cadastro de Empresa Contratante possibilita o cadastramento específico de cada empresa, assim como o módulo Cadastro de Município realiza o cadastramento dos municípios atendidos pelo Serviço de Saúde Ocupacional.

Esses dados cadastrados ficam arquivados sob forma de um banco de dados. A finalidade é deixar à disposição da equipe de Saúde Ocupacional as informações concernentes às atividades da empresa e do município.

O módulo SINAN - Sistema de Informação de Agravos de Notificação possibilita a notificação e o acompanhamento dos casos de acidente com material biológico.

A interface Notificação Individual (Figura 3), viabiliza as informações de identificação pessoal e profissional do funcionário vítima de acidente com material biológico. Isto acontecerá a partir da escolha do nome do funcionário (previamente cadastrado no módulo Cadastro de Funcionário) em uma lista apresentada por meio de um toque no dispositivo de entrada (mouse) sobre o campo nome. A seguir, deve ser acionado o ícone salvar do Menu para o arquivamento dos dados descritos.

Figura 3: Tela inicial do Módulo SINAN - Notificação Individual. Software para organização das informações de um serviço de Saúde Ocupacional. Caranduva, SP, 2010.

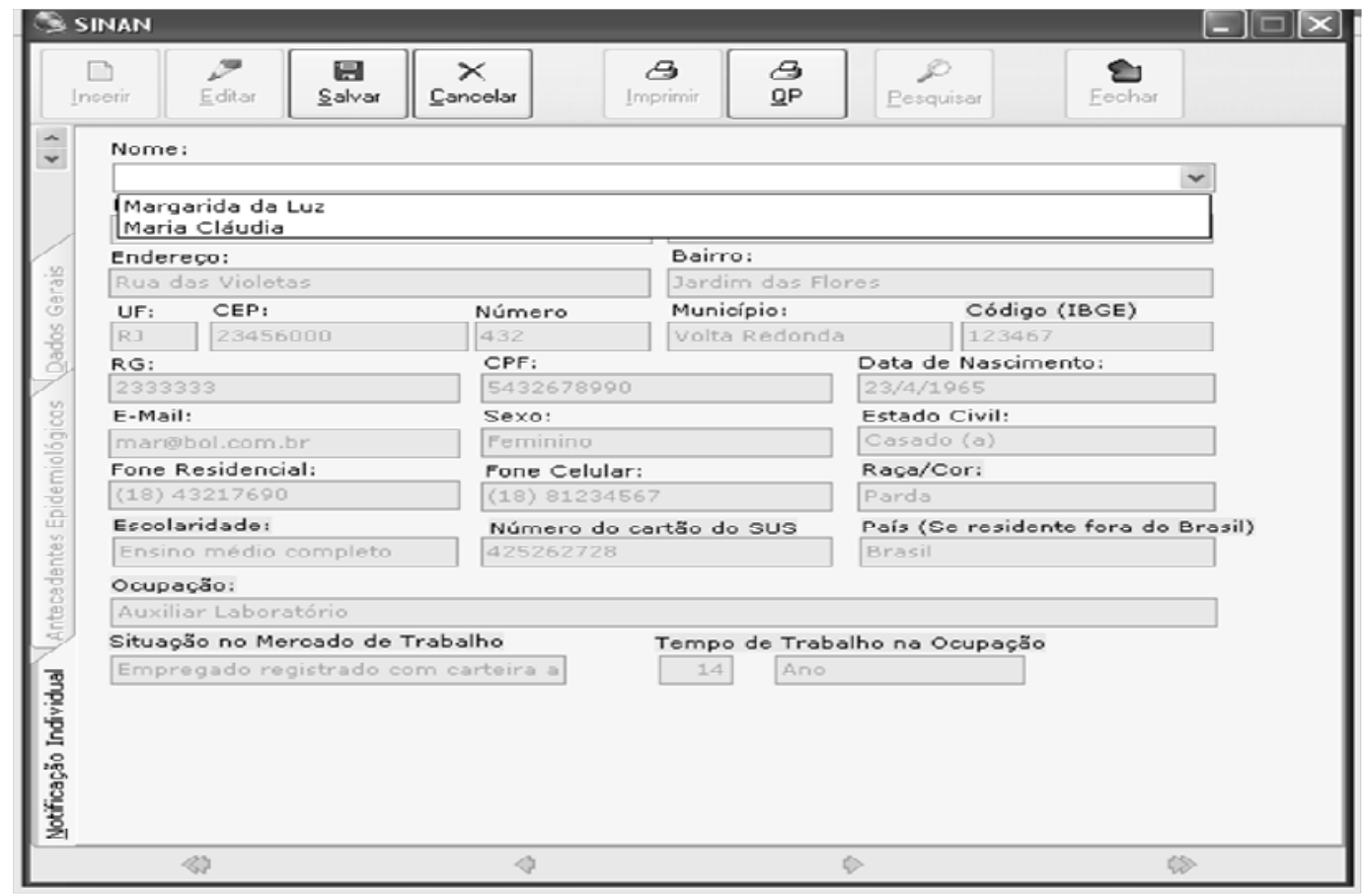

A interface, Antecedentes Epidemiológicos, viabiliza as informações de identificação da empresa e município onde ocorreu o acidente com material biológico. Isto acontecerá a partir da escolha do nome da empresa (previamente cadastrado no módulo Cadastro de Empresa Contratante), em uma lista apresentada por meio de um toque no dispositivo de entrada (mouse) sobre o campo nome. Após a inserção dos dados em todos os campos torna-se necessário acionar o ícone salvar do Menu principal.

A interface Dados Gerais tem por função cadastrar os dados referentes ao acidente de trabalho com material biológico propriamente dito.

Os dados nos campos código, agravo/doença, código CID e tipo de notificação são previamente inseridos em virtude da padronização pelo Ministério da Saúde (MS). 
No campo relativo ao número do COREN, deverá ser digitado o número de inscrição no Conselho Regional de Enfermagem do profissional enfermeiro responsável pela notificação do caso.

Conforme características observadas nas fichas manuais de notificações de acidentes utilizada pelo Serviço de Saúde Ocupacional, o campo - uso de EPI (Equipamento de Proteção Individual), foi elaborado na forma de múltipla escolha.

Para minimizar as atividades de digitação, os campos envolvendo data dispõem de um calendário que permite a adição dos dados com um toque no dispositivo de entrada (mouse) e os campos: unidade de saúde, tipo de exposição, material orgânico, agente, circunstância do acidente, situação vacinal e pedidos de exames também poderão ser cadastrados com um toque no dispositivo de entrada (mouse). É necessário salvar os dados inseridos, acionar o ícone
Imprimir e selecionar a opção 3, para que seja impresso

o Termo de Autorização para Coleta de Exames

\section{Laboratoriais.}

Ainda nessa tela, se houver recusa do funcionário acidentado em realizar o procedimento padrão de coleta de exames laboratoriais registrada nos campos de pedidos de exames, deverá ser acionado o ícone Imprimir, e o sistema gerará automaticamente o Termo de Responsabilidade - Recusa do Procedimento Padrão.

A interface Dados Gerais 2 (Figura 4), cadastra os dados referentes à fonte e aos testes sorológicos. Após salvar estes dados e acionar o ícone QP (Quimioprofilaxia), o sistema processa as informações que possibilitarão a análise situacional automática do caso. Será gerada, assim, a conduta a ser seguida pelo profissional enfermeiro em determinado acidente, identificada no sistema por Receita.

Figura 4: Tela Dados gerais 2. Software para organização

das informações de um serviço de Saúde Ocupacional. Caranduva, SP, 2010.

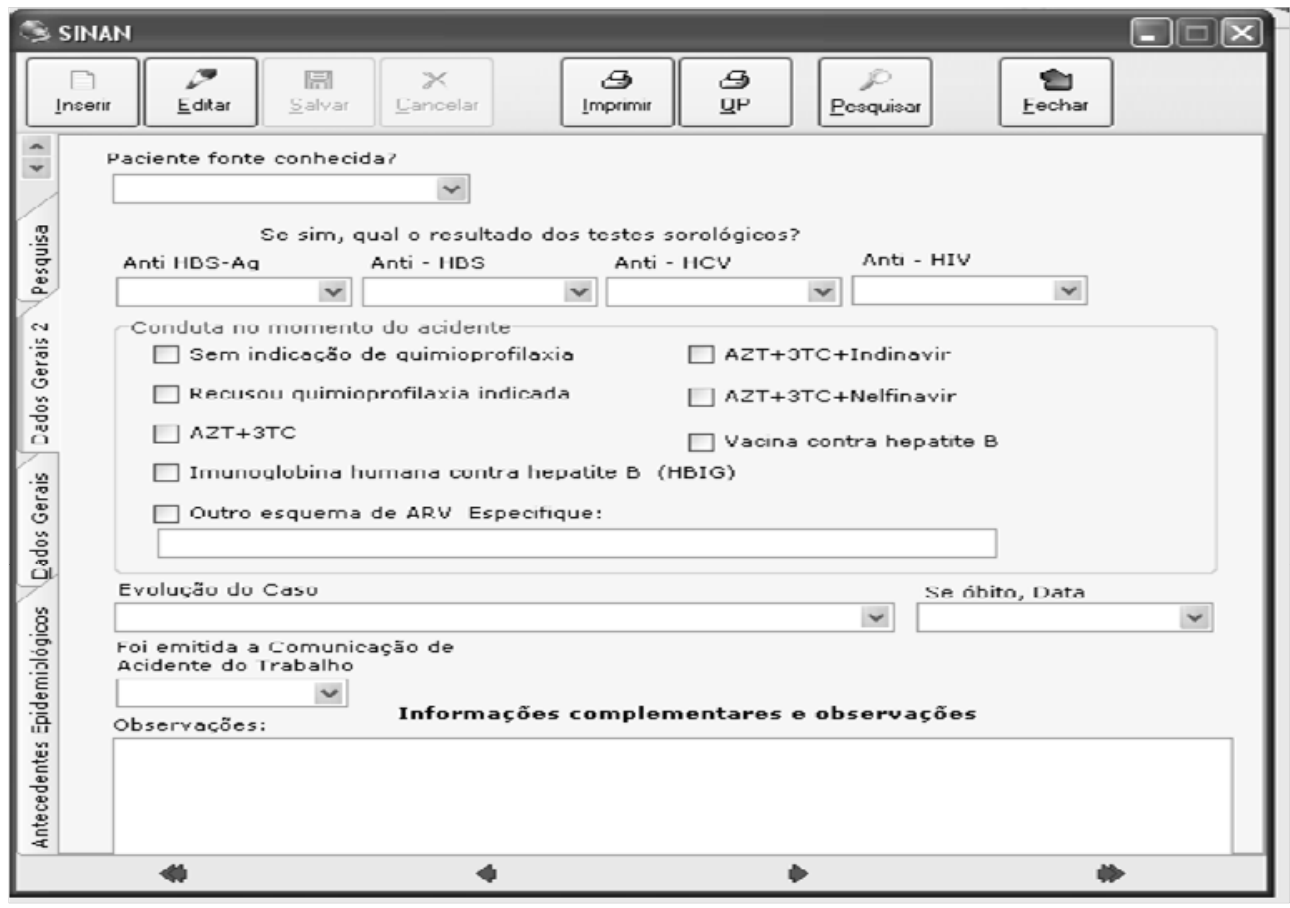

O campo conduta no momento do acidente foi elaborado sob a forma de múltipla escolha e deverá conter os dados de conduta contidos na Receita.

A interface Receita possui três ícones à esquerda da tela que permitirão ao usuário realizar alterações, salvar os dados atualizados e solicitar impressão do documento.

Ao término do processo de notificação, deverão ser salvos os dados inseridos em todos os campos $\mathrm{e}$ acionado o ícone Imprimir que apresentará as opções: 1 (Frente) e $\mathbf{2}$ (Verso), para impressão de todas as informações e posterior arquivo manual no prontuário em papel do funcionário, como rotina institucional.

Nessa mesma tela, registram-se também os dados de acompanhamento e desfecho do caso. Os dados referentes à evolução do caso, óbito e emissão de CAT (Comunicação de Acidente de Trabalho) podem ser 
inseridos com um toque no dispositivo de entrada (mouse).

As informações complementares e observações relativas aos casos são registradas em um campo com entrada de texto livre.

A interface, Pesquisa do módulo SINAN, permite acessar as informações cadastradas com relação a todas as notificações realizadas por meio de dois toques no dispositivo de entrada (mouse) sobre a escolha do nome do funcionário e data do acidente com material biológico. Essas informações são recuperadas em uma lista ao acessar o ícone pesquisar ou pela digitação dos caracteres de nome ou data da notificação nos respectivos campos dispostos na região inferior da tela. Essa dinâmica torna ágil a busca por informações, minimiza as atividades de digitação do usuário e possibilita maior controle sobre o acompanhamento dos casos.

\section{DISCUSSÃO}

Refletindo sobre a realidade encontrada no cenário em estudo, reconhece-se a necessidade de uma ferramenta computacional que proporcione a organização da informação; a otimização do tempo; a facilidade dos registros e de suas avaliações estatísticas como também o auxílio na tomada de decisão e acompanhamento dos casos.

Em se tratando das atividades assistenciais, relativas ao acidente com material biológico, o que se observa é a presença de lacunas de informação deixadas pelos profissionais enfermeiros, acarretando possíveis prejuízos à qualidade da assistência prestada. Isto ocorre porque os dados são coletados, mas dificilmente analisados de forma a gerar informação e conhecimento que sejam utilizados para desenhar, redirecionar ou tornar visível o atendimento prestado. A qualidade na automação e na velocidade da informação facilita e estabelece o diferencial no atendimento e no processo de tomada de decisão ${ }^{(15)}$.

Diante desse cenário, o desafio foi desenvolver um software que corresponda às necessidades específicas do profissional enfermeiro que atua em Saúde Ocupacional e atenda a expectativa de reduzir o tempo despendido na busca de informações por meio de um registro manual, minimizando os erros cometidos nas decisões baseadas em fluxogramas padronizados com interpretação realizada individualmente.

A criação e implementação de um software decorre da crescente necessidade organizacional de integrar funções e processos para melhorar o controle, a coordenação, e a capacidade de resposta da organização, permitindo que dados e informações fluam livremente entre diferentes setores ${ }^{(12)}$.

As dificuldades em termos de padronização do vocabulário de enfermagem e o fato de que as ações, muitas vezes, são planejadas de forma mais intuitiva, fazem com que o enfermeiro pouco explore os sistemas de informação para descrever suas práticas ${ }^{(15)}$.

Boa parte dos erros que ocorrem nos processos decisórios é em decorrência às falhas de informação ou de formação. Cometem-se erros por não estar devidamente preparado para analisar as situações complexas, para fazer escolhas e tomar decisões de modo a percorrer todas as alternativas possíveis para resolução dos problemas. O erro, muitas vezes pode ser fatal para nós e para os outros envolvidos na situação(16).

A melhoria da tomada de decisão depende da melhoria nos processos de controle e comunicação. Permitir acesso rápido à informação pode favorecer ações eficientes, maior controle e proporcionar diferentes informações necessárias ao desenvolvimento eficaz das atividades.

Sob esse contexto, buscou-se organizar toda a informação produzida no Serviço de Saúde Ocupacional em relação aos acidentes de trabalho com material biológico e torná-la acessível aos enfermeiros por meio do desenvolvimento de um sistema informatizado, com a finalidade de apoiar as decisões e as ações de enfermagem nessas circunstâncias.

\section{CONCLUSÕES}

O produto desta pesquisa culminou no desenvolvimento de um software com a finalidade de organizar e apoiar a tomada de decisão frente às informações acerca dos acidentes de trabalho com material biológico.

A elaboração de um sistema de registro informatizado foi estruturada por meio de um processo complexo, com alguns desafios a serem vencidos. Acredita-se que o maior deles foi a busca e a interpretação de todas as informações geradas em decorrência do acidente e a compreensão da metodologia de análise situacional para a tomada de decisão.

O caminho metodológico permitiu uma melhor compreensão da rotina do serviço e facilitou a identificação dos usuários do sistema, as demandas, as necessidades e as fontes de informação, assim como o 
delineamento do ambiente informacional e sua automatização.

Houve uma constante preocupação em desenhar o software de modo a disponibilizar o máximo de informações com um fácil acesso a todos os usuários. Foi possível realçar o uso da tecnologia computacional para gerenciar um grande número de informações e apresentar um fortalecimento para a combinação das mesmas, obtendo como resultado a decisão "exata" a cada caso de acidente ocorrido.

\section{REFERÊNCIAS}

1. Benito CAV, Licheski AP. Sistemas de Informação apoiando a gestão do trabalho em saúde. Rev Bras Enferm [Internet]. 2009 [cited 2011 set 30];62(3):447-50. Available from: http://www.scielo.br/pdf/reben/v62n3/18.pdf.

2. Évora YDM. A enfermagem na era da informática. Rev. Eletr. Enf. [Internet] 2007 [cited 2011 set 30];9(1):14. Available from: http://www.fen.ufg.br/revista/v9/n1/v9n1a01.htm.

3. Vieira M, Padilha MI, Pinheiro RDC. Análise dos acidentes com material biológico em trabalhadores da saúde. Rev Lat Am Enfermagem [Internet]. 2011 [cited 2011 set 30];19(2):332-9. Available from: http://www.scielo.br/pdf/rlae/v19n2/pt 15.pdf.

4. Cardoso ACM, Figueiredo RM. Situações de risco biológico presentes na assistência de enfermagem nas unidades de saúde da família (USF). Rev Lat Am Enfermagem. [Internet]. 2010 [cited 2011 set 30];18(3):368-72. Availale from: http://www.scielo.br/pdf/rlae/v18n3/pt_11.pdf.

5. Caetano JA, Soares E, Braquehais AR, Rolim KAC. Acidentes de trabalho com material biológico no cotidiano da enfermagem em unidade de alta complexidade. Enferm. glob. [Internet]. 2006 [cited 2011 set 30];5(9). Available from: http://revistas.um.es/eglobal/article/view/371/363.

6. Marziale $\mathrm{MH}$, Zapparoli AS, Felli VE, Anabuki MH. Rede de Prevenção de Acidentes de Trabalho: uma estratégia de ensino a distância. Rev Bras Enferm. 2010[ cited 2011 set 30];63(2):2506. Available from: http://www.scielo.br/pdf/reben/v63n2/13.pdf. 7. Chiodi MB, Marziale MHP, Robazzi MLCC. Acidentes de trabalho com material biológico entre trabalhadores de unidades de saúde pública. Rev Lat Am Enfermagem. [Internet]. 2007 [cited 2011 set 30]; 15(4):632-8. Available from: http://www.scielo.br/pdf/rlae/v15n4/pt_v15n4a17.pdf.

8. Palomares MLE, Marques IR. Contribuições dos sistemas computacionais na implantação da Sistematização da Assistência de Enfermagem. J. health inform. [Internet] 2010 [cited 2011 set 30];2(3):78-82. Available from: http://www.jhi-sbis.saude.ws/ojsihi/index.php?journal $=$ jhi-

sbis\&page $=$ article\&op =view\&path $\% 5 B \% 5 D=94 \&$ path $\% 5 B \% 5 D=34$. 9. Aguiar RV, Cassiani SHDB. Desenvolvimento e avaliação de ambiente virtual de aprendizagem em curso profissionalizante de enfermagem. Rev Lat Am Enfermagem. [Internet]. 2007 [cited 2011 set 30];15(6):1086-91. Available from: http://www.scielo.br/pdf/rlae/v15n6/pt 04.pdf.

10. Rangel AL, Évora YDM. Elaboração automática da escala periódica de trabalho dos profissionais de enfermagem por meio de um software específico. Rev. Eletr. Enf. [Internet] 2007 [cited 2011 set 30];9(1):17-30. Available from: http://www.fen.ufg.br/revista/v9/n1/v9n1a02.htm.

11. Sperandio DJ, Évora YDM. Planejamento da assistência de enfermagem: proposta de um software-protótipo. Rev Lat Am Enfermagem. [Internet]. 2005 [cited 2011 set 30];13(6):937-43. Available from: http://www.scielo.br/pdf/rlae/v13n6/v13n6a04.pdf.

12. Laudon KC, Laudon JP. Sistemas de Informação gerenciais: administrando a empresa digital. Marques AS, tradutor. 5th ed. São Paulo. Prentice Hall; 2004.
Uma das prioridades exigidas foi que o sistema reduzisse, consideravelmente, o tempo despendido com a documentação de informações, facilitando a entrada de dados com um toque no dispositivo mouse e diminuindo as inserções digitadas por meio do teclado.

Acredita-se que esse produto inovador auxiliará o enfermeiro na tomada de decisão e facilitará o planejamento e acompanhamento de enfermagem nos casos de acidente com material biológico até o seu desfecho.

13. Pressman RS. Engenharia de software. Santos JCB, tradutor. 3rd ed. São Paulo: Makron Books; 2006.

14. Ministério da Saúde; Conselho Nacional de Saúde. Resolução No 196/96 - Normas regulamentadoras de pesquisa envolvendo seres humanos. Brasília (Brasil): Ministério da Saúde; 1996.

15. Silveira DT, Marin HF. Conjunto de Dados Mínimos de Enfermagem: construindo um modelo em sáude ocupacional. Acta paul. enferm. [Internet]. 2006 [cited 2011 set 30];19(2):218-27. Available from: http://www.scielo.br/pdf/ape/v19n2/a15v19n2.pdf.

16. Ciampone MHT, Melleiro MM. O Planejamento e o processo decisório como instrumentos do processo de trabalho gerencial. In: Kurgant P. Gerenciamento em enfermagem. 1st ed. Rio de Janeiro. Guanabara Koogan; 2005. cap. 4. p. 37-53.

Artigo recebido em 27.09.2010.

Aprovado para publicação em 05.09.2011.

Artigo publicado em 30.09.2011. 